\title{
A Multi-Touch Alignment Guide for Interactive Displays
}

\author{
Mathias Frisch, Ricardo Langner, Sebastian Kleinau, Raimund Dachselt \\ User Interface \& Software Engineering Group \\ Otto-von-Guericke-University Magdeburg, Germany \\ \{mfrisch, dachselt\}@ovgu.de, \{rlangner, sebastian.kleinau\}@st.ovgu.de
}

\begin{abstract}
Precise alignment of graphical objects and creating proper layouts is crucial in many domains, such as graphic design or graph editing. In this paper we are presenting a multitouch alignment guide for interactive displays. It allows adjusting the alignment and spacing of graphical objects by multi-touch input and bimanual interaction.
\end{abstract}

ACM Classification: H5.2 [Information interfaces and presentation]: User Interfaces. - Graphical user interfaces.

\section{General terms: Design}

Keywords: Multi-Touch, Interactive Surfaces, Alignment

\section{INTRODUCTION}

Accurate positioning of graphical objects is an essential activity in many applications. For example, in digital design tools graphics or textboxes have to be arranged in a precise way or in diagram editors nodes of graphs have to be properly aligned. Usually these tasks are accomplished by invoking functions from menus (e.g., for right alignment) or by entering values (e.g., for setting the spacing between items). Beyond that, snap-dragging [1] or guides are used to align objects along straight lines.

However, these approaches have several drawbacks. For example, manually entered parameters have to be changed several times until a pleasant result is obtained. Furthermore, creating more complex arrangements with different distances between objects (Fig. 1 right) can be tedious.

Interactive displays are promising for graphic applications $[2,3]$ and to overcome these disadvantages. Multi-touch and pen gestures allow quick mode switches and a more natural and direct interaction. Many tasks can be performed simultaneously by both hands. However, creating precise layouts with these modalities is still challenging.

In this paper we present work in progress concerning a multi-touch alignment guide - a layout and alignment tool for multi-touch enabled displays. It allows the arrangement of objects and adjusting parameters, such as spacing, in a precise and interactive way by multi-touch input.

Permission to make digital or hard copies of all or part of this work for personal or classroom use is granted without fee provided that copies are not made or distributed for profit or commercial advantage and that copies bear this notice and the full citation on the first page. To copy otherwise, to republish, to post on servers or to redistribute to lists, requires prior specific permission and/or a fee.

ITS'10, November 7-10, 2010, Saarbrücken, Germany.

Copyright 2010 ACM 978-1-4503-0399-6/10/11...\$10.00.

\section{RELATED WORK}

Several works such as [4] are dealing with techniques for rotating and translating digital objects by multi-touch interaction. However, these works focus on precise positioning of single objects. They do not consider alignment tasks such as adjusting the spacing between several objects. In order to align objects or to create symmetric shapes, several graphics applications integrate ruler tools (e.g., [6]). Usually they are designed for single point input. An exception is the work of Raisamo et al. [5]. Here bimanual interaction techniques by mouse and trackball are investigated. We seize on these approaches but investigate how multi-touch input can be applied for aligning graphical objects.

\section{THE MULTI-TOUCH ALIGNMENT GUIDE}

A multi-touch alignment guide consists of two parts: a straight line and a handle. The line is used to bind graphical objects, such as pictures or nodes of graphs. Bound objects stick to the guide when it is moved. The handle can be applied to reposition the guide and to switch modes. In particular, it consists of three areas: two for rotation at both ends and one for translation at its center (Fig. 2, left). Free rotation and translation is achieved by touching both rotation areas and moving the handle with two fingers (Fig. 1, left). Thereby, the handle adjusts its size according to the distance of the fingers. Touching the handle with one finger constrains the interaction to translation (Fig. 1, right) or rotation depending on the touched area.

\section{Creating and deleting guides}

Multi-touch alignment guides can be created in different ways. A guide appears when a stroke is drawn. Thereby, the length of the guide corresponds to the length of the sketched stroke. Beyond that, holding two fingers on the canvas results in a guide with infinite length. We also suggest dragging guides from the edge onto the display, as known from state-of-the-art graphics editing applications.

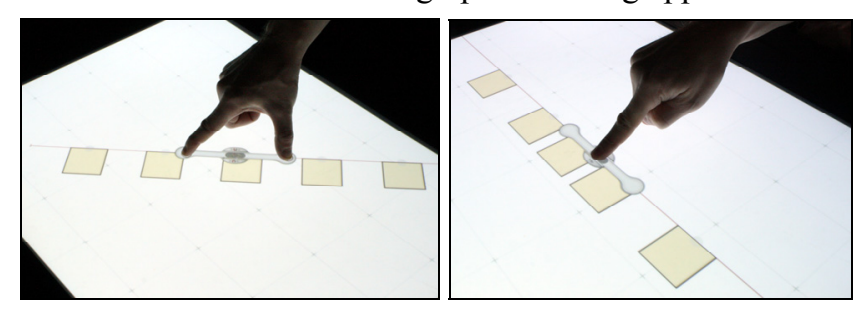

Figure 1: A handle is used to reposition the guide and the bound objects. It can be moved freely with two fingers (left); one finger constrains it to translation (right) or rotation. 


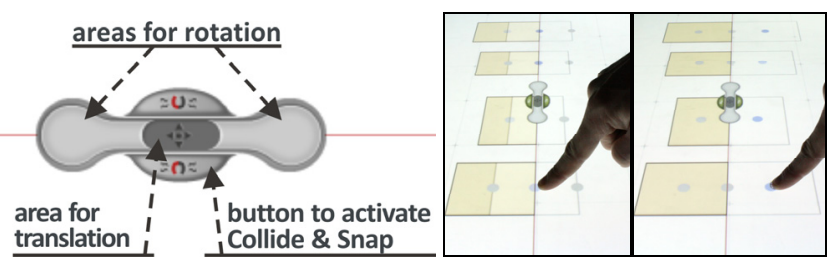

Figure 2: Structure of the handle (left), changing alignment by dragging an object across the guide with "all"-mode activated (right).

Furthermore, if touch and pen input is combined $[2,3]$ the pen could be laid down on the display to create a guide. Guides can be deleted by dragging them off-screen or by performing a wipe gesture across them.

\section{Binding and Releasing Objects}

For binding objects to the guide, we implemented two different interaction techniques.

\section{Flick \& Snap}

Initially the guide is set to the Flick \& Snap mode. Thus, the guide can be translated and rotated freely without affecting any objects on the canvas. Binding a graphical object is achieved by flicking the object towards the guide. As a result the object is moved to the guide in an animated way and is bound at the location where the animation path intersects the guide. In that way a quick and explicit assignment of objects to several guides is possible by flicking in the respective direction.

\section{Collide \& Snap}

When Collide \& Snap is activated objects are automatically bound if they collide with the guide. In that way, users can move the guide across the canvas and "collect" objects.

Switching between the two modes is achieved by pressing a button located at the handle. Buttons are placed on both sides of the handle to provide interaction from each direction (Fig.2 left). The mode switch is possible during movement, thus providing a smooth way of interaction without interrupting the workflow for toggling the button. In both modes objects can be released by dragging or flicking them away from the guide.

\section{Positioning objects}

For adjusting their positions, bound objects are manipulated directly by touch input. Thereby, the guide serves as a constraint and restricts the movement of the objects. A button at the handle is used to specify if a gesture affects just one object (the one which is touched) or all bound objects ("all"-mode). For that, we did not introduce an additional button. Instead, the buttons for activating Collide \& Snap change their functionality when the handle is released. This is possible as moving the guide and positioning bound objects cannot be done simultaneously.

\section{Adjusting the alignment}

Dragging objects across the guide results in centering objects on the guide or aligning them to one side of the guide. During the drag gesture feedback is given by previews

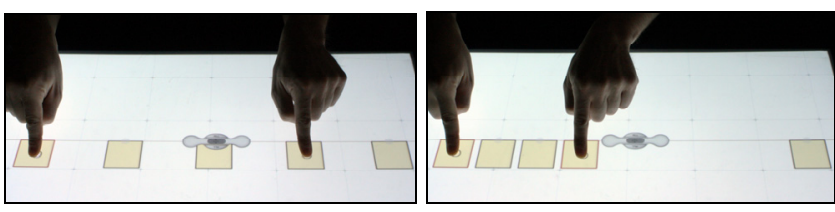

Figure 3: Holding one object and dragging another one adjusts the spacing of the objects in between.

(Fig. 2, right). Objects snap to the respective position when the finger is lifted. In Figure 2 all bound objects are affected, as the "all"-mode is activated.

\section{Adjusting the spacing}

Dragging one object moves it along the guide. If the "all"mode is activated and an object is dragged, the other bound objects are translated as well. Thereby, the ratio of their spacing is maintained. A similar effect is achieved by holding an object and dragging another one with a second finger. As a result, all objects in between are pushed together (Fig. 3) or moved apart. Furthermore, performing a "shake"-gesture on the handle (moving it quickly back and forth) results in distributing the bound objects equally.

\section{CONCLUSION AND FUTURE WORK}

We presented a multi-touch alignment guide for interactive displays. It allows binding, rearranging and adjusting the spacing of graphical objects. For future work we plan to add scaling or mirroring bound objects and combining several guides with each other. Beyond that, we will consider other shapes, such as circles, rectangles or free drawn curves. Finally, user studies shall be conducted to compare our approach with existing techniques.

Acknowledgements. This work was partly funded by the "Stifterverband für die Deutsche Wissenschaft" from funds of the Claussen-Simon-Endowment.

\section{REFERENCES}

1. Bier, E. A. and Stone, M. C. 1986. Snap-dragging. SIGGRAPH Comput. Graph. 20, 4 (Aug. '86), 233-240.

2. Frisch, M., Heydekorn, J., Dachselt, R. 2009. Investigating Multi-Touch and Pen Gestures for Diagram Editing on Interactive Surfaces. In Proc. of IST '09, 167-174.

3. Hinckley, K., Yatani, K., Pahud, M., Coddington, N., Rodenhouse, J., Wilson, A., Benko, H., and Buxton, B. 2010. Manual deskterity: an exploration of simultaneous pen+touch direct input. In Proc. of CHI EA '10, 2793-2802.

4. Nacenta, M. A., Baudisch, P., Benko, H., and Wilson, A. 2009. Separability of spatial manipulations in multitouch interfaces. In Proc. of Graphics interface 2009 175-182.

5. Raisamo, R. 1999. An alternative way of drawing. In Proc. of CHI '09, pp. 175-182.

6. Zeleznik, R. C., Bragdon, A., Liu, C., and Forsberg, A. 2008. Lineogrammer: creating diagrams by drawing. In Proc. of UIST '08, 161-170. 